NOTE

\title{
Novel phycodnavirus genes amplified from Canadian freshwater environments
}

\author{
Steven M. Short ${ }^{1,2, *}$, Oksana Rusanova ${ }^{2}$, Michael A. Staniewski ${ }^{1}$ \\ ${ }^{1}$ University of Toronto Mississauga, Department of Biology, Mississauga, Ontario L5L 1C6, Canada \\ ${ }^{2}$ University of Toronto, Department of Ecology and Evolutionary Biology, Toronto, Ontario M5S 3B2, Canada
}

\begin{abstract}
Extant and newly designed primers for the polymerase chain reaction (PCR) were used to amplify phycodnavirus DNA polymerase ( $\mathrm{polB}$ ) gene fragments from numerous samples collected at different times of the year from 3 freshwater environments in Ontario, Canada. Overall, a total of 143 cloned PCR fragments were sequenced and 106 putative phycodnavirus polB gene fragments were identified. Although most of these 106 gene fragments were very closely related (i.e. $>97 \%$ identical) to $p o l B$ sequences from chloroviruses, or environmental sequences related to prasinoviruses, 16 represented 2 new types of phycodnavirus polB genes. More specifically, polB fragments that formed a new clade of chloroviruses were amplified from Lake Ontario using newly designed Chlorovirus-specific PCR primers, and a polB sequence most closely related to genes from the prymnesioviruses PgV-03T and CbV-PW1 was amplified from a pond sample from Mississauga, Ontario, using the degenerate algal virus-specific PCR primers AVS1 and AVS2. Thus, the results of the present study provide evidence for a new type of Chlorovirus, and the first observation of polB sequences from freshwater phycodnaviruses that are presumed to infect algae other than chlorophytes.
\end{abstract}

KEY WORDS: Phycodnaviruses · Algal viruses $\cdot$ Chlorella viruses · Prymnesioviruses · Freshwater · DNA polymerase $\cdot$ polB

\section{INTRODUCTION}

Phycodnaviruses are a remarkable group of viruses because they are closely related to the largest known viruses, the Mimiviridae (Monier et al. 2008), they have large, diverse genomes (Dunigan et al. 2006, Iyer et al. 2006), they encode surprising biochemical pathways (e.g. Markine-Goriaynoff et al. 2004, Wilson et al. 2005, Vardi et al. 2009), and they infect one of the most ecologically important groups of organisms on Earth, eukaryotic algae. To study the genetic richness of these viruses in the environment, researchers have designed polymerase chain reaction (PCR) primers to target phycodnavirus B-family DNA polymerase genes $(p o l B)$ (Chen \& Suttle 1995), and the major capsid protein (Larsen et al. 2008). Although neither primer set is truly universal for the Phycodnaviridae, each has the ability to amplify its targets from several phycodnavirus taxa
(Larsen et al. 2008). For example, the algal virusspecific polB primers AVS1 and AVS2 have been used to amplify genes from different types of chloroviruses, prasinoviruses and prymnesioviruses (Chen \& Suttle 1995, Brussaard et al. 2004, Bellec et al. 2009). However, with the exception of 1 operational taxonomic unit (OTU, or a DNA sequence $\leq 97 \%$ identical to any other) from the Gulf of Mexico (Chen et al. 1996), and 2 from Kane'ohe Bay, O'ahu, Hawai'i, USA (Culley et al. 2009), polB fragments amplified from natural environments using the AVS primers have all been most closely related to cultivated marine prasinoviruses (Short \& Short 2008, Clasen \& Suttle 2009, Culley et al. 2009).

Considering that the only known freshwater phycodnaviruses are chloroviruses, the fact that all phycodnavirus polB sequences amplified from freshwater environments have been most closely related to Prasinovirus genes is somewhat puzzling (Short \& Short 
2008, Clasen \& Suttle 2009). To resolve this issue and to determine whether other types of phycodnavirus could be PCR-amplified from freshwater environments, 2 complementary research strategies were employed. First, new group- and taxon-specific PCR primers were designed to amplify polB fragments from chloroviruses, and second, samples collected from different locations during different seasons were surveyed.

\section{MATERIALS AND METHODS}

At various times of the year during the ice-free period between 2007 and 2009, surface water samples of 11 were collected from several freshwater habitats in Ontario, Canada, including Lake Ontario $\left(43^{\circ} 32.614^{\prime} \mathrm{N}, 79^{\circ} 34.995^{\prime} \mathrm{W}\right)$, a storm water pond on the University of Toronto Mississauga (UTM) campus in Mississauga $\left(43^{\circ} 32.759^{\prime} \mathrm{N}, 79^{\circ} 39.574^{\prime} \mathrm{W}\right)$, and Crawford Lake $\left(43^{\circ} 28.140^{\prime} \mathrm{N}, 7^{\circ} 56.954^{\prime} \mathrm{W}\right)$. Following collection, all samples were immediately transported back to the laboratory for processing as described in Short \& Short (2008) except that all samples collected after 2007 were filtered through $47 \mathrm{~mm}$ diameter $0.45 \mu \mathrm{m}$ pore size Durapore ${ }^{\circledR}$ PVDF membrane filters of (Millipore) only, and samples collected in 2009 were centrifuged at $118000 \times g$ for $3.5 \mathrm{~h}$ in an SW32Ti rotor (Beckman Coulter). Samples collected in 2007 were pre-filtered through $47 \mathrm{~mm}$ diameter GC50 glass-fibre filters (Advantec MFS), and samples collected before 2009 were centrifuged using an SW40Ti rotor (Beckman). The pre-filtration step was abandoned because it was not necessary for the small volumes $(<200 \mathrm{ml})$ that were processed, and the centrifugation procedure was changed to increase sample throughput. Neither of these changes would negatively affect the virus preparations because it has been shown that filtration through glass-fibre filters can actually cause loss of virus particles (Wommack et al. 2010), and the calculated time to pellet phycodnaviruses with the SW32Ti rotor is ca. $3.3 \mathrm{~h}$ if a very conservative sedimentation coefficient of $65 \mathrm{~S}$ is assumed for phycodnaviruses; sedimentation coefficients range from 42 to $>1000 \mathrm{~S}$ for all known viruses (Lawrence \& Steward 2010). Following centrifugation, the supernatant was decanted, and pelleted material was resuspended and subjected to a freeze/thaw treatment as described in Short \& Short (2008).

PCR primer sets specifically targeting the chloroviruses ATCV-1, CVM-1 and PBCV-1, as well as a degenerate primer set targeting all three (CHLVd) (Table 1) were designed from aligned Chlorovirus polB genes (Fig. 1). The efficacy of these newly designed primers was determined by amplifying gene fragments from filtered $(47 \mathrm{~mm}$ diameter, $0.45 \mu \mathrm{m}$ pore size Durapore ${ }^{\circledR}$ PVDF), freeze/thawtreated cellular lysates from the viruses PBCV-1, CVM-1 and ATCV-1 grown on Chlorella NC64a, Chlorella Pbi and Chlorella SAG 3.83, respectively. The Chlorovirus-specific primers and the algal virusspecific primers AVS1 and AVS2 (Chen \& Suttle 1995) were used to PCR-amplify gene fragments from a variety of freeze/thaw-treated environmental samples. All PCR reactions, and the cloning and sequencing methods, were conducted as described in Short \& Short (2008), except that the annealing temperatures for PCR with the ATCVs, CVMs, PBCVs and CHLVd primer sets were $48,50,44$ and $52^{\circ} \mathrm{C}$, respectively, and a C1000 ${ }^{\mathrm{TM}}$ thermal cycler (Bio-Rad Laboratories) was used. Each $50 \mu \mathrm{l}$ PCR reaction was electrophoresed in $1.5 \%$ LE agarose (Promega) gels in $1 \mathrm{X}$ TAE (40 mM Tris, $20 \mathrm{mM}$ acetic acid, $1.0 \mathrm{mM}$ EDTA, $\mathrm{pH}$ 7.6), and gels were stained in a $0.5 \mu \mathrm{g} \mathrm{ml}^{-1}$ ethidium bromide solution and visualized on a Molecular Imager ${ }^{\circledR}$ ChemiDoc ${ }^{\mathrm{TM}}$ XRS system (Bio-Rad Laboratories). Amplified fragments of the appropriate size (i.e. as noted in Table 1) were excised from the gels, extracted using a QIAquick Gel Extraction kit (Qiagen), and cloned using pGEM ${ }^{\circledR}-\mathrm{T}$ Vector System II (Promega) as described in Short \& Short (2008). Plasmid inserts were sequenced at The Centre for Applied Genomics (TCAG) at The Hospital for Sick Children, Toronto, Ontario, Canada. Only full-length

Table 1. Newly designed Chlorovirus-specific DNA polymerase (polB) PCR primers

\begin{tabular}{|c|c|c|c|}
\hline $\begin{array}{l}\text { Primer } \\
\text { name }\end{array}$ & $\begin{array}{l}\text { Virus targeted/expected } \\
\text { amplicon size (bp) }\end{array}$ & Forward primer ${ }^{\mathrm{a}, \mathrm{b}}$ & Reverse primer ${ }^{a, b}$ \\
\hline ATCVs & ATCV-1/610 & AAG AAA GGT GCC TAC TTT GAA C & AGG TCG TTC GGA GCT TTG TAC T \\
\hline CVMs & CVM-1/645 & AAG AAG GGA GCA TAC TTC ACG C & CAA AAT GTA AGG GTA ATA GAT CTT C \\
\hline PBCVs & PBCV-1/600 & CTT ATC GCA GCT CTC GAT TTT G & GTT CGG TGC TCG GAA ATC CTT C \\
\hline CHLVd & $\begin{array}{r}\text { PBCV-1, ATCV-1, } \\
\text { CVM-1/560-575 }\end{array}$ & CCW ATC GCA GCW CTM GAT TTT G & ATC TCV CCB GCV ARC CAC TT \\
\hline
\end{tabular}




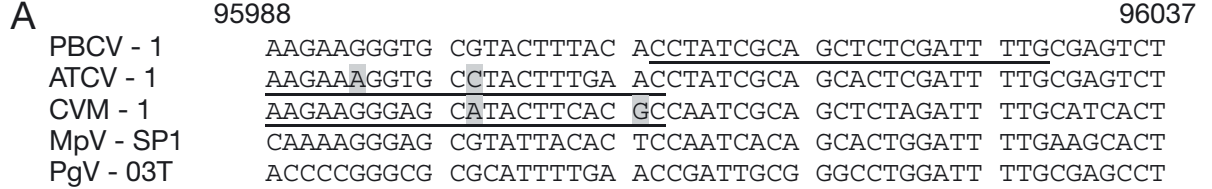

\section{Forward specific primer}

96647

PBCV -1
ATCV - 1
CVM - 1
MpV - SP1
PgV - 03T

CAATCCCCGC TCGCTGTTTT TTGAACTCAC CTCCAAGTCG TTCGGTGCTC GGAAATCCTT CGTAATCTCC CAAGATGTAC GGGTAGTAAA TCTTCTCAAA CTCCAGGTCG TTCGGAGCTT TGTACTCCTT TGTGATCTCG CAAAATGTAA GGGTAATAGA TCTTCTCAAA CTCCAAATCG TTGGGCGCCC GATACTCCTT TGTAATCTCA TAGAAAGTAA GGCCAATATA CCTTTTCTAA CTCCAAATTG TTTGGCTTCT TGAATAGGGC ACTACATTCT CAGAATAAAC GGATACATCA CTTTTTCAAA TTCCAGTTCA ATCGGTTTTT TAAAGGTCGC GCTAATACGA

\section{Reverse specific primer}

96578 


\section{RESULTS AND DISCUSSION}

Because previous reports have already demonstrated that the primers AVS1 and AVS2 can be used to amplify phycodnavirus genes from freshwater environments (Short \& Short 2008, Clasen \& Suttle 2009), only 2 samples were amplified with these primers. These samples were collected from Lake Ontario, Canada, on July 16, 2008, and the UTM pond on September 18, 2009, and were amplified, cloned, and sequenced as components of other ongoing studies in our laboratory. Interestingly, 2 polB fragments that were not putative Prasinovirus genes were amplified using AVS1 and AVS2; prior to this study all freshwater sequences amplified with these primers have been closely related to marine Prasinovirus polB genes. The gene fragment LO16jul08.23 was nearly identical (98\% identical over 689 nucleotides) to polB of the Chlorovirus FR483, and was obtained from 36 cloned fragments (generated by PCR) of a July 16, 2008, Lake Ontario sample (Fig. 2); the other 35 sequences from that clone library were all putative Prasinovirus gene

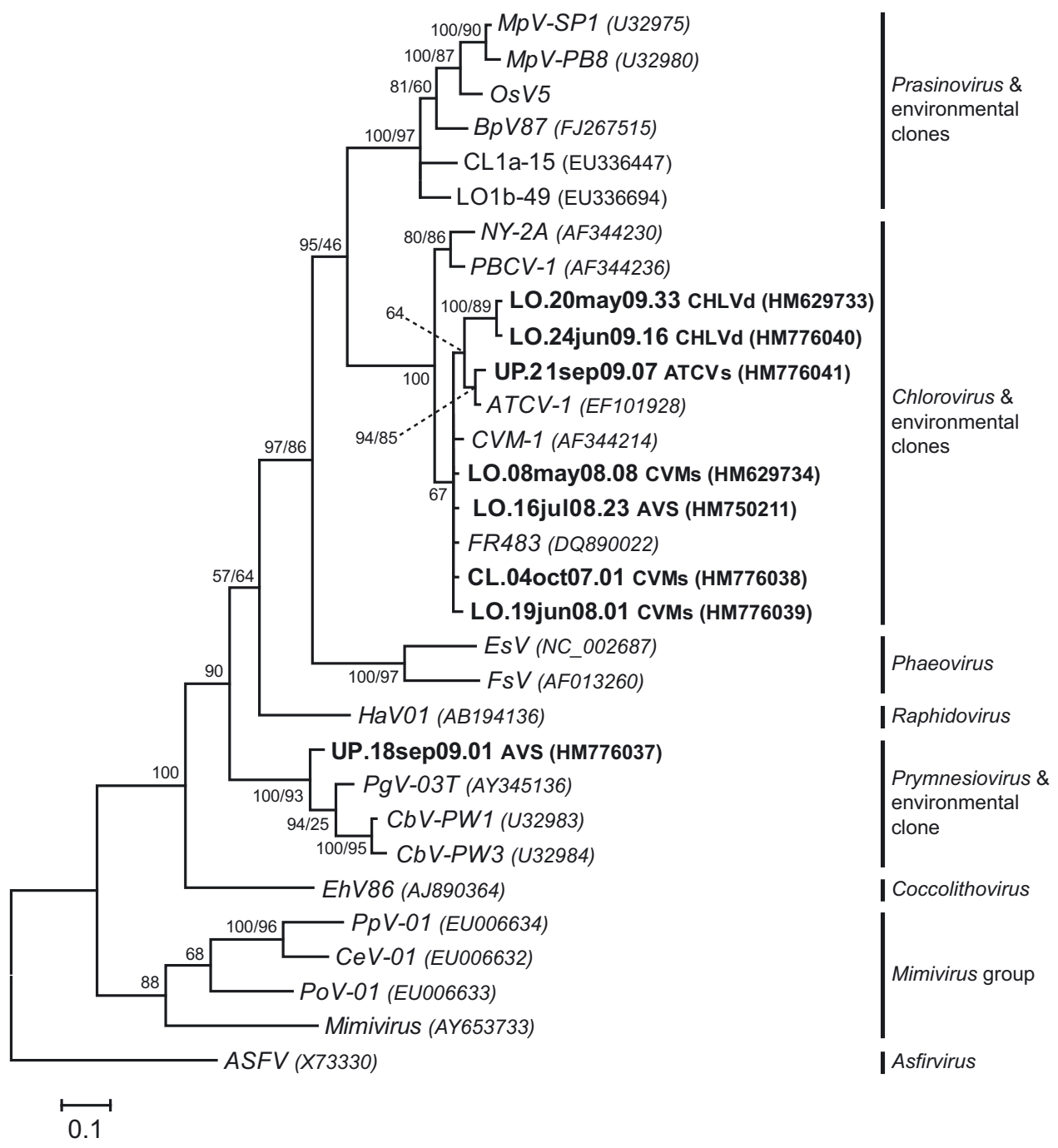

Fig. 2. Phylogenetic tree of phycodnaviruses and other nucleo-cytoplasmic large DNA viruses based on Bayesian inference (BI) of inferred amino acid sequences from partial DNA polymerase genes. Where the maximum likelihood (ML) tree topology matched the BI topology, numbers at branch nodes indicate the posterior probability followed by the aLRT (approximate likelihood ratio test) branch support from SH-like (Shimodaira-Hasegawa-like) values. Where the topology varied between BI and ML trees, only posterior probability values are shown. Sequences obtained in this study are in bold; sequences from cultivated viruses and other environmental clones are in italics and normal type, respectively. Names of the sequences obtained in this study include their origin (Lake Ontario: LO; Crawford Lake: CL; or the University of Toronto Mississauga pond: UP), date of sample collection (as day/month/year), an arbitrary clone number, and the PCR primer set used to obtain the sequence. GenBank accession numbers are listed in parentheses beside each sequence, and clusters containing sequences from cultivated viruses are indicated to the right of the tree and are labelled with the virus genera. Scale bar indicates the proportion of changes per site 
fragments (Short et al. in press). Additionally, the gene fragment UP.18sep09.01 was amplified from a UTM pond sample collected on September 18, 2009, and was most closely related to polB genes from prymnesioviruses that infect Phaeocystis globosa and Chrysochromulina brevifilum (Fig. 2). Furthermore, this putative Prymnesiovirus polB fragment was the only sequence recovered from the September 18, 2009, UTM pond sample; all 13 cloned fragments that were sequenced were identical.

When testing the newly designed Chlorovirus genespecific PCR primer sets ATCVs, CVMs and PBCVs on virus lysates, each primer set amplified DNA fragments from their target viruses only. For example, the CVMs primers amplified gene fragments from cellular lysates of CVM-1, but not from lysates of PBCV-1 or ATCV-1. In contrast, the Chlorovirus-specific degenerate primers were able to amplify DNA fragments of the expected size from all 3 of the Chlorovirus lysates tested. Because no other phycodnavirus isolates were available at the time of this study, it was not possible to determine whether the Chlorovirus-specific degenerate primers could amplify polB genes from other phycodnaviruses. However, as these degenerate primers have 11 mismatches when compared to the polB sequences of the virus MpV-SPI (Fig. 1), and, with respect to their polB genes, prasinoviruses such as MpV-SP1 are the closest relatives of chloroviruses (Fig. 2), it seems unlikely that they could amplify any genes other than Chlorovirus polB. Moreover, the fact that all identifiable sequences obtained with the degenerate primers were putative Chlorovirus polB genes suggests that these newly designed degenerate primers were specific for chloroviruses. PCR of environmental samples using the Chlorovirus-specific primers CVMs and ATCVs produced amplicons of the expected size only, whereas the primers PBCVs and degenerate Chlorovirus-specific primers (CHLVd) produced the expected amplicons as well as fragments that did not correspond to the expected size (Table 2).

PCR reactions with all Chlorovirus-specific primers except PBCVs produced amplified polB gene fragments that clustered among known phycodnaviruses (Fig. 2, Table 2). Overall, only 57 polB sequences were obtained from 94 cloned fragments that were originally amplified with the Chlorovirus gene-specific and degenerate PCR primers (Table 2). Without exception, sequences from the other 37 cloned fragments were unidentifiable (i.e. the sequencing reactions failed, or BLAST comparisons to the GenBank nucleotide collection revealed no significant homologies). PCR with the ATCVs primers resulted in the successful amplification of a polB gene fragment from only a single sample collected from the UTM pond on September 21, 2009. In contrast, reactions with the CVMs primers led to the amplification of polB gene fragments from many samples collected at various times of the year from all 3 freshwater locations (Table 2). Although reactions with the PBCVs primers produced amplicons of the expected size for several samples, no polB sequences were recovered from any cloned PBCVs amplicons (Table 2). Because sequences related to PBCV-1 were not recovered with any phycodnavirus primers used in this study, it seems very unlikely that the PBCVs amplicons were bona fide Chlorovirus genes. In fact, it is plausible that viruses related to PBCV-1 were not present or were below the level of detection in the samples tested, or that PBCVs primers are not useful for obtaining environmental Chlorovirus sequences. Although the disappointing results with the PBCVs primers highlight challenges associated with PCR primer design and amplification of environmental samples, the recovery of Chlorovirus polB fragments via PCR with the other Chlorovirus gene-specific primers demonstrates the utility of virus-specific primers for environmental surveys. Furthermore, sequences obtained with the CVMs primers have already been useful for developing quantitative molecular methods for assessing virus dynamics in situ (Short et al. in press).

For many samples, reactions with the primers CHLVd produced amplicons of the expected size. However, these primers also produced nonspecific amplicons from almost every sample, suggesting that the reaction conditions could not be made stringent enough to prevent spurious amplification (Table 2); increasing the stringency of PCR with CHLVd always resulted in failed amplification. Nonetheless, of the 33 CHLVd cloned fragments that were sequenced, 24 were identified as putative Chlorovirus polB fragments; 9 could not be identified because they shared no significant homology to any sequences in GenBank. Of these 24 polB fragments, 21 were $>97 \%$ identical to each other and to polB sequences from viruses infecting Chlorella Pbi (e.g. FR483 and CVM-1). The other 3 polB fragments amplified with CHLVd were considered novel Chlorovirus gene fragments and were recovered from the May 20, 2009, and June 24, 2009, Lake Ontario samples. Two of these fragments (LO.20may09.33 and LO.24jun09.16) are shown in Fig. 2 to highlight the fact that they were obtained from more than 1 sample, but all 3 were $>97 \%$ identical to each other. These particular polB fragments were considered novel because they clustered among known chloroviruses but formed their own distinct clade (Fig. 2). Also, nucleotide BLAST comparisons of the novel sequences to the GenBank nucleotide collection (nr/nt database) revealed that the top hits for LO.20may09.33 and LO.24jun09.16 were DNA polymerase genes of PBCV-1 and NY-2C, respectively, each with only $74 \%$ sequence identity over the entire 
Table 2. PCR and sequencing results for samples targeted with Chlorovirus-specific primers. UTM: University of Toronto Mississauga. -: no amplification; +: correct size product; ns: non-specific amplification

\begin{tabular}{|c|c|c|c|c|c|}
\hline \multirow{2}{*}{ Sample date } & \multirow{2}{*}{ Sample location } & \multicolumn{4}{|c|}{ Amplification results (no. of polB sequences/no. of plasmids sequenced) } \\
\hline & & CVMs & ATCVs & PBCVs & CHLVd \\
\hline $27 \mathrm{Sep} 07$ & Lake Ontario & - & - & $+\mathrm{ns}(0 / 4)$ & $+\mathrm{ns}$ \\
\hline 4 Oct 07 & Crawford Lake & $+(4 / 5)$ & - & $+\mathrm{ns}$ & $+\mathrm{ns}$ \\
\hline 10 Oct 07 & Lake Ontario & $+(9 / 10)$ & - & $+\mathrm{ns}$ & $+\mathrm{ns}$ \\
\hline 8 May 08 & Lake Ontario & $+(8 / 10)$ & - & ns & $+\mathrm{ns}(5 / 5)$ \\
\hline 5 Jun 08 & Lake Ontario & $+(2 / 2)$ & - & ns & $+\mathrm{ns}$ \\
\hline 19 Jun 08 & Lake Ontario & $+(4 / 5)$ & - & ns & ns \\
\hline 14 Aug 08 & Lake Ontario & - & - & ns & ns \\
\hline 9 Oct 08 & Lake Ontario & - & - & ns & ns \\
\hline 22 Jan 09 & Lake Ontario & - & - & ns & $+\mathrm{ns}$ \\
\hline 4 May 09 & UTM pond & $+(2 / 2)$ & - & ns & $+\mathrm{ns}$ \\
\hline 20 May 09 & Lake Ontario & + & - & ns & + ns $(4 / 5)$ \\
\hline 2 Jun 09 & UTM pond & $+(3 / 3)$ & - & $+\mathrm{ns}(0 / 2)$ & + ns $(5 / 13)$ \\
\hline 9 Jun 09 & Lake Ontario & + & - & ns & $+\mathrm{ns}$ \\
\hline 9 Jun 09 & UTM pond & + & - & ns & $+\mathrm{ns}(5 / 5)$ \\
\hline 24 Jun 09 & UTM pond & + & - & ns & ns \\
\hline 24 Jun 09 & Lake Ontario & + & - & $+\mathrm{ns}(0 / 5)$ & $+\mathrm{ns}(5 / 5)$ \\
\hline 23 Jul 09 & Lake Ontario & + & - & ns & - \\
\hline 23 Jul 09 & UTM pond & - & - & $+\mathrm{ns}(0 / 5)$ & ns \\
\hline 21 Sep 09 & Lake Ontario & - & - & $+\mathrm{ns}$ & $+\mathrm{ns}$ \\
\hline 21 Sep 09 & UTM pond & - & $+(1 / 8)$ & $\mathrm{ns}$ & + \\
\hline
\end{tabular}

query length (578 nucleotides). Although the BLAST results seem to suggest that the novel sequences were more closely related to PBCV-1 than ATCV-1, the phylogeny based on 83 confidently aligned amino acids placed the novel Chlorovirus fragments in a group nearest to ATCV-1 (Fig. 2). However, because the sequences used for the BLAST searches and the phylogenetic analysis are different (nucleotides versus informative amino acid positions), these results are not contradictory and highlight the fact that LO.20may09.33 and LO.24jun09.16 represent a unique, novel group of Lake Ontario chloroviruses.

The results of the present study reinforce and extend previous studies demonstrating that Canadian freshwaters are home to diverse phycodnaviruses (Short \& Short 2008, Clasen \& Suttle 2009) by reporting sequences that fall outside previously observed clusters of environmental phycodnavirus polB sequences (e.g. Chen et al. 1996, Short \& Suttle 2002, Short \& Short 2008, Clasen \& Suttle 2009, Culley et al. 2009), and by demonstrating the existence of polB fragments from unknown types of phycodnaviruses. Amplification with degenerate Chlorovirus-specific primers led to the recovery of Lake Ontario sequences that represent a new type of Chlorovirus. The notion that the CHLVd amplicons LO.20may09.33 and LO.24jun09.16 represent unknown chloroviruses is supported by the reasoning of Clasen \& Suttle (2009) because the genetic distances between these amplicons and the polB genes of cultivated chloroviruses are greater than the distances between different types of cultivated chloroviruses (e.g. ATCV-1 and CVM-1), or different viruses within the genera Prasinovirus (Bellec et al. 2009), or Prymnesiovirus (Brussaard et al. 2004) (Fig. 2). Similar reasoning can be applied to the UP.18sep09.01 AVS fragment to support its identification as a novel Prymnesiovirus gene.

Although the hosts for the viruses that encode the novel Chlorovirus-like polB gene fragments are unknown, numerous free-living Chlorella spp. have been observed in past phycological surveys of Lake Ontario (Munawar \& Munawar 1996), and gene fragments closely related to Chlorella spp. psbA have recently been amplified from Lake Ontario (e.g. GenBank accession numbers HM629672, HM629554 and HM629626). Thus, the coincidence of potential hosts and host sequences in the same environment permits speculation that the viruses encoding the novel Chlorovirus-like polB sequences infect free-living Chlorella-like algae, and supports their putative identification as chloroviruses. Similarly, a gene fragment (GenBank accession number HQ112284) most closely related to $p s b A$ from prymnesiophyte algae such as Emiliania huxleyi, Chrysochromulina polylepis and Prymnesium parvum was amplified from the same UTM pond sample from which the putative Prymnesiovirus fragment UP.18sep09.01 originated. Again, this coincidence supports speculation that the UP.18sep09.01 fragment originated from a novel type of Prymnesiovirus. The discovery of a polB sequence closely related to Prymnesiovirus genes provides the first evidence that the host range of freshwater phy- 
codnaviruses extends beyond chlorophyte algae, and the discovery of new Chlorovirus sequences demonstrates that new types of chloroviruses await isolation.

Acknowledgements. We thank C. Short and N. Musrap for their assistance with sample collection and processing. Chlorovirus isolates and Chlorella cell cultures were generously provided by J. L. Van Etten. This work was supported by the Canadian Foundation for Innovation Leaders Opportunity Fund and NSERC Discovery grants awarded to S.M.S

\section{LITERATURE CITED}

Bellec L, Grimsley N, Moreau H, Desdevises Y (2009) Phylogenetic analysis of new prasinoviruses (Phycodnaviridae) that infect the green unicellular algae Ostreococcus, Bathycoccus and Micromonas. Environ Microbiol Rep 1: 114-123

Brussaard CPD, Short SM, Frederickson CM, Suttle CA (2004) Isolation and phylogenetic analysis of novel viruses infecting the phytoplankton Phaeocystis globosa (Prymnesiophyceae). Appl Environ Microbiol 70:3700-3705

Castresana J (2000) Selection of conserved blocks from multiple alignments for their use in phylogenetic analysis. Mol Biol Evol 17:540-552

Chen F, Suttle CA (1995) Amplification of DNA-polymerase gene fragments from viruses infecting microalgae. Appl Environ Microbiol 61:1274-1278

> Chen F, Suttle CA, Short SM (1996) Genetic diversity in marine algal virus communities as revealed by sequence analysis of DNA polymerase genes. Appl Environ Microbiol 62:2869-2874

Clasen JL, Suttle CA (2009) Identification of freshwater Phycodnaviridae and their potential phytoplankton hosts, using DNA pol sequence fragments and a genetic-distance analysis. Appl Environ Microbiol 75:991-997

Culley AI, Asuncion BF, Steward GF (2009) Detection of inteins among diverse DNA polymerase genes of uncultivated members of the Phycodnaviridae. ISME J 3: 409-418

Dereeper A, Guignon V, Blanc G, Audic S and others (2008) Phylogeny.fr: robust phylogenetic analysis for the nonspecialist. Nucleic Acids Res 36:W465-W469

Dunigan DD, Fitzgerald LA, Van Etten JL (2006) Phycodnaviruses: a peek at genetic diversity. Virus Res 117: $119-132$

Edgar RC (2004) MUSCLE: multiple sequence alignment with high accuracy and high throughput. Nucleic Acids Res 32: 1792-1797

Guindon S, Gascuel O (2003) A simple, fast, and accurate

Editorial responsibility: Paul del Giorgio,

Montreal, Canada algorithm to estimate large phylogenies by maximum likelihood. Syst Biol 52:696-704

Iyer LM, Balaji S, Koonin EV, Aravind L (2006) Evolutionary genomics of nucleo-cytoplasmic large DNA viruses. Virus Res 117:156-184

Larsen JB, Larsen A, Bratbak G, Sandaa RA (2008) Phylogenetic analysis of members of the Phycodnaviridae virus family using amplified fragments of the major capsid protein gene. Appl Environ Microbiol 74:3048-3057

Lawrence JE, Steward GF (2010) Purification of viruses by centrifugation. In: Wilhelm SW, Weinbauer MG, Suttle CA (eds) Manual of aquatic viral ecology. American Society of Limnology and Oceanography, Waco, TX, p 166-181

> Markine-Goriaynoff N, Gillet L, Van Etten JL, Korres H, Verma N, Vanderplasschen A (2004) Glycosyltransferases encoded by viruses. J Gen Virol 85:2741-2754

Monier A, Larsen JB, Sandaa RA, Bratbak G, Claverie JM, Ogata $H$ (2008) Marine mimivirus relatives are probably large algal viruses. Virol J 5:12

Munawar M, Munawar IF (1996) Phytoplankton dynamics in the North American great lakes, Vol 1: Lake Ontario, Erie and St. Clair. SPB Academic Publishing, Amsterdam

Ronquist F, Huelsenbeck JP (2003) MrBayes 3: Bayesian phylogenetic inference under mixed models. Bioinformatics 19:1572-1574

Short CM, Rusanova O, Short SM (in press) Quantification of virus genes provides evidence for seed-bank populations of phycodnaviruses in Lake Ontario, Canada. ISME J doi:10.1038/ismej.2010.183

Short SM, Short CM (2008) Diversity of algal viruses in various North American freshwater environments. Aquat Microb Ecol 51:13-21

Short SM, Suttle CA (2002) Sequence analysis of marine virus communities reveals that groups of related algal viruses are widely distributed in nature. Appl Environ Microbiol 68:1290-1296

Tamura K, Dudley J, Nei M, Kumar S (2007) MEGA4: molecular evolutionary genetics analysis (MEGA) software version 4.0. Mol Biol Evol 24:1596-1599

Vardi A, Van Mooy BAS, Fredricks HF, Popendorf KJ, Ossolinski JE, Haramaty L, Bidle KD (2009) Viral glycosphingolipids induce lytic infection and cell death in marine phytoplankton. Science 326:861-865

Wilson WH, Schroeder DC, Allen MJ, Holden MTG and others (2005) Complete genome sequence and lytic phase transcription profile of a Coccolithovirus. Science 309: 1090-1092

Wommack KE, Sime-Ngando T, Winget DM, Jamindar S, Helton RR (2010) Filtration-based methods for the collection of viral concentrates from large water samples. In: Wilhelm SW, Weinbauer MG, Suttle CA (eds) Manual of aquatic viral ecology. American Society of Limnology and Oceanography, Waco, TX, p 110-117

Submitted: August 9, 2010; Accepted: November 24, 2010 Proofs received from author(s): February 7, 2011 